\title{
Personalized Toe Spreaders with Three-Dimensional Scanning and Printing for Hallux Valgus
}

\author{
Yong Ho Cha ${ }^{1}$, Keun Ho Lee ${ }^{1}$, Dong Kyu Kim¹, Sung Jae Kim², Sang Jun Kim ${ }^{1}$ \\ ${ }^{1}$ Department of Physical and Rehabilitation Medicine, Samsung Medical Center \\ Gangnamgu Irwonro 50th, Seoul, Republic of Korea \\ ${ }^{2}$ Department of Medical Device Management \& Research, SAIHST, Sungkyunkwan University \\ yh78.cha@samsung.com; kh96.lee@samsung.com; mrdkim03@naver.com; sungjaewkd@naver.com; \\ guitarren.kim@samsung.com
}

\begin{abstract}
The purpose of this study is to produce personalized toe spreaders for hallux valgus through 3D printing and 3D scanning techniques. Eight patients (14 feet) with hallux valgus participated in our study and were scanned with the 3D scanner. After designing toe spreaders with the modelling program, we made toe spreaders with 3D printing technique in a selective laser sintering method. Hallux valgus angle (HVA) and intermetatarsal angle (IMA) were compared before and after wearing. Changes of HVA and IMA after wearing the toe spreader were significantly different $(P<0.001$ and $P=0.034)$. Total amount of time for making a customized toe spreader was approximately 7 hours and total price was approximately 50 dollars. Personalized toe spreaders for hallux valgus made with 3D scanning and 3D printing techniques improved HVA and IMA. This suggests the possibility of the commercial use of 3D printed customized toe spreaders for hallux valgus.
\end{abstract}

Keywords: Hallux valgus; three dimensional; personalized; toe spreader

\section{Introduction}

Hallux valgus is a foot deformity which is characterized by lateral deviation of the great toe and medial deviation of the first metatarsal, and its treatment consists of use of toe spreaders, valgus splints, and operative correction. Toe spreader is a simple, cheap, and easy to wear orthosis, and can reduce the pain caused by the bunion. Toe spreader can be worn everyday if it fits into the toe space of patients, can provide comfort to the patients, and can correct the toe axis during wearing. However, the ready-made silicone toe spreader does not fit accurately into the toe space of a particular patient, and it takes time to make and deliver the custom made toe spreader to the patients. In addition, the custom made toe spreader is expensive because of customization and needs to be reconstructed from the beginning if it is destroyed or needs correction because the structural frames of a toe spreader are not stored.

Three-dimensional (3D) printers are also utilized in various medical fields such as Department of Stomatology, Orthopaedic, and Rehabilitation Medicine. Dentists apply 3D printing techniques to manufacture dental plaster casts [1]. Orthopaedic surgeons use 3D printers to simulate the artificial hip joint [2]. Physiatrists use 3D printers to make individual foot insoles [3]. In addition to this, recent researches suggest the possibility of creating artificial organs including ear [4]. Compared to the traditional alternatives, the advantage of 3D printing technique is that the 3D printed products can be personalized, modified, and reproduced more easily, which can lower the price of personalized products.

Therefore, it will be useful to create a personalized toe spreader using a 3D printer because this toe spreader will be personalized, easily modifiable, and reproducible at low price. The purpose of this study is to produce personalized toe spreaders for hallux valgus through 3D printing and 3D scanning techniques. Then, we will use these toe spreaders in hallux valgus patients and evaluate their functionality.

\section{Methods}

We recruited volunteers who complained of toe deformity and visited our outpatient department from February 2015 to May 2015. Among them, we selected 8 patients who were diagnosed with hallux valgus. Hallux valgus was diagnosed when the hallux valgus angle (HVA) was less than $160^{\circ}$ according to the previous article [5]. One patient had hallux valgus 
on his left foot, one patient had hallux valgus on the right foot, and 6 patients had hallux valgus on both feet. All patients had bunions on the first metatarsal head.

Two kinds of 3D scanners were used in this study; Eva (Artec ${ }^{\mathrm{TM}}$ Eva, Artec Group, Luxembourg) and Spider (Artec ${ }^{\mathrm{TM}}$ Spider, Artec Group, Luxembourg) for scanning of feet. Eva and Spider have a similar function with a video camera in terms of real-time capturing the objects. The scanner captures up to 16 frames per second and these frames are aligned automatically in real-time, which make scanning easy and fast. Because of the 3D resolution up to $0.5 \mathrm{~mm}$, Eva's textured models have been used in diverse industries, such as, computer graphic/animation, forensics, and medicine. Spider is designed to scan small objects with complex geometry, sharp edges and thin ribs. Therefore, Spider is usually used to scan small and complex objects, such as, human ear, keys, or coins.

Scanning process was conducted at our outpatient department. The 3D scanner could not detect the interdigital space because the interdigital space was shadowed; therefore, we inserted therapy putty (Sammons Preston ${ }^{\circledR}$, Patterson Medical Holdings Inc., Illinois, USA) into the interdigital space between the first and second toes and scanned the patients' feet in the inserted state with Eva (figure 1). The size of the putty was modified till the axis of first toe was less than $10^{\circ}$ along with the axis of the first metatarsus. After extracting the putty from the foot, we scanned the molded putty again with Spider (figure 1).

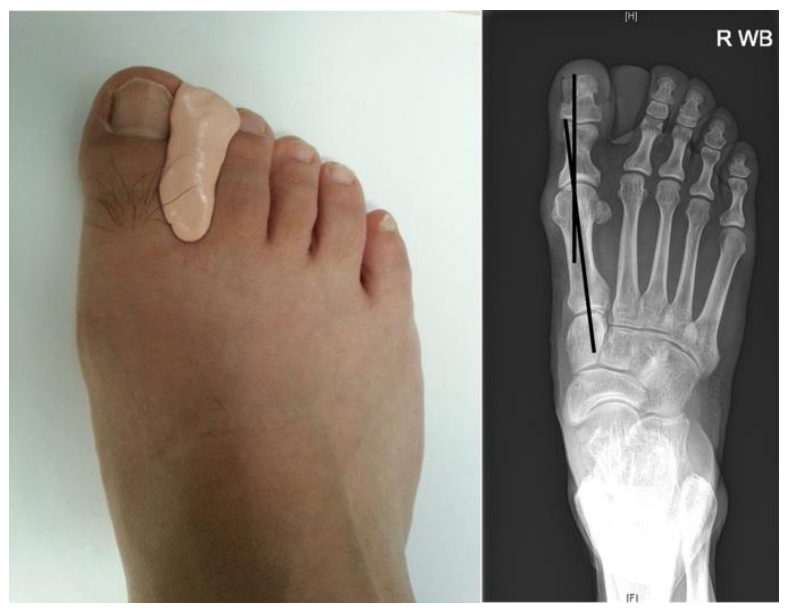

Fig. 1: Therapy putty was inserted for scanning the interdigital space. The size of the putty was modified till the axis of first toe was less than $10^{\circ}$ along with the axis of the first metatarsus.

Using these scanned files, we designed toe spreaders with the modeling program (Geomagic Freeform, 3D SYSTEMS, SC, USA). This modeling program uses 'virtual clay' to provide unparalleled creative freeform, and includes a phantom device; a true 3D interface with force feedback. At this stage, we designed a toe spreader for each patient considering the shape of his/her foot (figure 2). The newly designed toe spreader consisted of two components, the separator part and the fixing part. The separator part had a role in separating the first and second toes to bring the axis of the first toe and the first metatarsal within $10^{\circ}$ range. The fixing part enveloped the first toe partially to inhibit displacement and rotation of the toe spreader.

Using these modeling files, the designed toe spreaders were printed using selective laser sintering (SLS) type 3D printer (Objet500 Connex1, Stratasys, USA). Printing process was conducted at the 3D Convergence Technology Center, Kyungpook National University. This printer offers 14 base materials including transparent materials with great dimensional stability and surface smoothness, rubber-like materials suitable for non-slip and non-scratch surfaces or simulated over molding, rigid opaque materials in white, gray and blue and black simulated polypropylene materials with toughness and durability to create living hinges, flexible closures and snap-fit prototypes. For preparation of toe spreaders, VeroClearRGD810 (Stratasys Ltd, Minnesota, USA) and TangoGray FLX950 (Stratasys Ltd, Minnesota, USA) were used. There was no post-processing after the toe spreader was printed out.

We received the personalized toe spreaders from the 3D Convergence Technology Center and applied them to the patients for one week and conducted the evaluation after one week (figure 2). For the evaluation, we used the Foot and Ankle Outcome Score (FAOS) [6] and User Satisfaction Survey (USS) [7]. In addition, simple radiographic anterior-posterior (AP) images 
of the foot in standing position were checked after wearing the toe spreaders for the measurement of HVA and intermetatarsal angle (IMA). We used Image J software (NIH, USA) for the measurement of HVA and IMA.

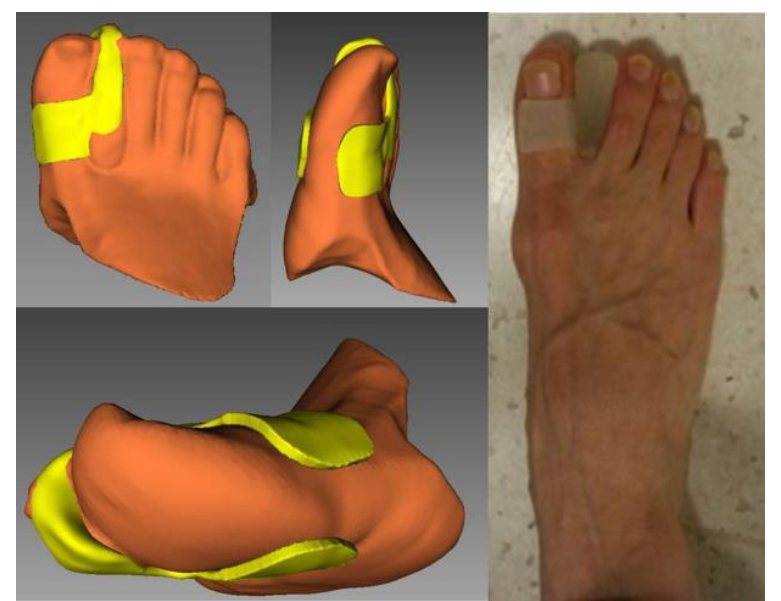

Fig. 2: Toe spreader was designed with the modeling program. The toe spreader consisted of the separator part and the fixing part. Using these modeling files, the designed toe spreader was printed using selective laser sintering (SLS) type 3D printer and applied.

For the statistical analysis, Paired- $t$ test was used to find the significant difference of HVA and IMA before and after the application of the toe spreader and Pearson correlation analysis was conducted to find the correlation between the initial severity of angles and their changes. We did not calculate the necessary sample size because this was a preliminary study. Statistical analyses were performed in SPSS 22.0 (IBM SPSS Statistics; IBM Corporation, Somers, NY).

Finally, for the safety of the 3D-printed materials we investigated the possible side effects in patients including skin rash, dermatitis, and skin ulcer.

Written informed consent was obtained from all of the participants, and this research was approved by the Institutional Review Board of our hospital (IRB No.2014-12-013).

\section{Results}

All the data (14 feet in 8 patients) were collected before and one week after the application of the toe spreaders. Initial HVA was $154.0^{\circ} \mathrm{SD} 8.8^{\circ}$ and it was changed to $160.5^{\circ} \mathrm{SD} 7.8^{\circ}$ after wearing toe spreaders, and this difference was statistically significant (Paired- $t=-5.263, P<0.001$ ). The IMA also changed from $16.5^{\circ} \mathrm{SD} 1.9^{\circ}$ to $17.7^{\circ} \mathrm{SD} 0.8^{\circ}$ after wearing the toe spreader (figure 3), and this difference was statistically significant (Paired- $t=-2.376, P=0.034$ ). When the changes in the HVA and IMA were assessed according to their initial severity, the changes in the angle were correlated with the initial severity (Pearson $r=-0.480, P=0.043$ for HVA, Pearson $r=-0.905, P<0.001$ for IMA) (figure 4).

HVA

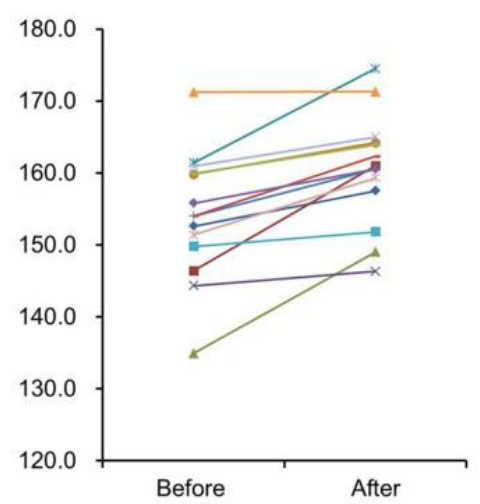

IMA

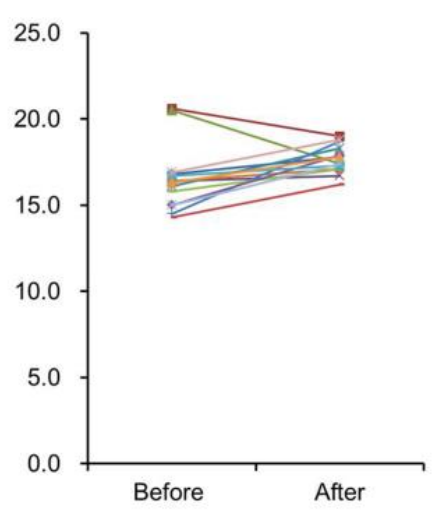

Fig. 3: Changes in the hallux valgus angle (HVA) (left) and intermetatarsal angle (IMA) (right) after wearing the toe spreader were presented in 14 feet. HVA and IMA changed significantly after wearing the toe spreader. 

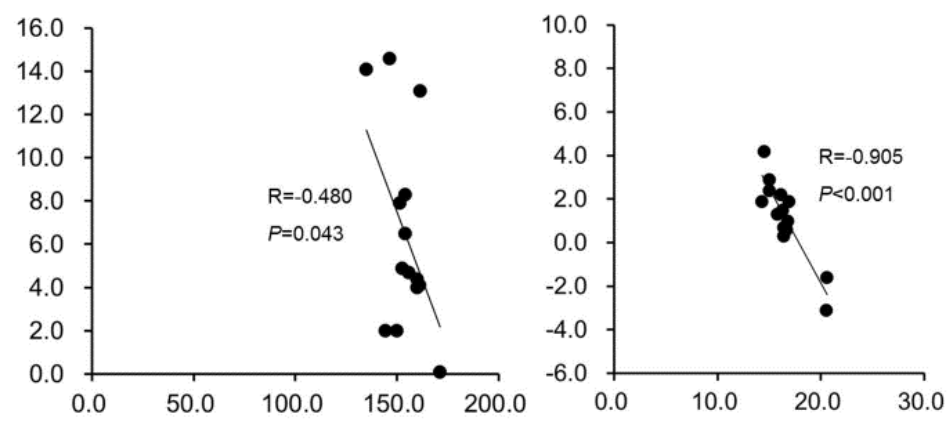

Fig. 4: Changes in the HVA (left) and IMA (right) were assessed according to their initial severity in 14 feet. The changes in the HVA and IMA were correlated with the initial severity.

\section{Discussion}

Our results were similar to that in the study by Tang [8], in which the HVA changed by $6.5^{\circ}$. Although this change was less than that after the surgical procedure; first metatarsal osteotomy which provides a correction of an average $20.1^{\circ}$ in a meta-analysis [9], all participants had an HVA more than $160^{\circ}$ except for one participant. The corrected HVA more than $160^{\circ}$ could improve the biomechanical parts of the body, which could prevent possible complications including bunions, osteoarthritis of the second metatarsophalangeal joint [10] and patellofemoral pain syndrome [11].

Average score on the USS was 7.4 SD 5.4, and it ranged from 2 to 18 points. Five participants were generally satisfied with the toe spreader, but the other participants were not satisfied with the toe spreader. All scores for sub-items of the FAOS except for quality of life were more than 50, which meant that all participants generally felt satisfied. Three participants reported poor quality of life after wearing the toe spreaders because they felt uncomfortable during activities. All data from the USS and FAOS are presented in table 1.

Table 1: Demographic data of patients and satisfaction scores.

\begin{tabular}{|c|c|c|c|c|c|c|c|c|}
\hline Patient & Sex & Age & $\mathrm{USS}^{*}$ & FAOS & & & & \\
\hline & & & & Other & Pain & $\overline{\mathrm{ADL}}$ & Sports & QoL \\
\hline JHK & $\mathrm{F}$ & 50 & 2 & 89.3 & 100 & 100 & 100 & 75 \\
\hline KIN & $F$ & 65 & 18 & 96.4 & 94.4 & 72.1 & 95 & 18.6 \\
\hline HSL & $\mathrm{F}$ & 62 & 7 & 92.9 & 100 & 100 & 100 & 100 \\
\hline KSJ & $F$ & 15 & 3 & 64.3 & 47.2 & 58.8 & 50 & 50 \\
\hline MOB & $\mathrm{F}$ & 55 & 7 & 89.3 & 80.6 & 97.1 & 100 & 43.8 \\
\hline JMY & $\mathrm{F}$ & 58 & 10 & 85.7 & 66.7 & 70.6 & 85 & 56.3 \\
\hline KKJ & $\mathrm{F}$ & 58 & 2 & 71.4 & 50 & 52.9 & 50 & 37.5 \\
\hline JMS & $\mathrm{F}$ & 50 & 10 & 100 & 100 & 53 & 50 & 56.3 \\
\hline
\end{tabular}

Total amount of time for making a customized toe spreader was approximately 7 hours and total price was approximately 50 dollars. There were no side effects including skin rash and dermatitis during the period of wearing the toe spreaders. 
There is one problem to overcome while using the 3D scanning and printing techniques for hallux valgus. We scanned participants' feet in a non-weight bearing position for scanning plantar areas of the foot. Considering that hallux valgus causes difficulty in walking, it would be better to design toe spreaders with the participants in standing position. Gong et al showed that the HVA decreased on weight-bearing position compared to non-weight-bearing position [12]. We think that toe spreaders would need to be thicker to correct the hallux valgus deformity if we had scanned the foot in a weight-bearing position.

The cost of our 3D printed toe spreader was approximately 50 dollars, which was priced between the ready-made silicone toe spreader (approximately 20 dollars) and the customized toe spreader (approximately 150 dollars) available in our country. However, because the ready-made silicone toe spreader does not fit the patient's toe space, so makes the patient uncomfortable, this personalized 3D printed toe spreader will be a good replacement in the market of orthosis. Considering that 3D printing materials are being developed and introduced rapidly, the cost of 3D printed materials will reduce soon.

\section{Conclusion}

The 3D printer is a revolutionary tool that will change many things in the medical field. Our study provides one example (orthosis for hallux valgus) of these forthcoming changes and suggests the possibility of commercial use of 3D printed customized toe spreaders for hallux valgus in terms of advantages of individualization, cost, and time.

\section{Acknowledgements}

This work was supported by the ICT R\&D program of MSIP/IITP. [B0101-14-1081, Development of ICT based software platform and service technologies for medical 3D printing applications].

\section{References}

[1] M. Kasparova, L. Grafova, P. Dvorak, et al., "Possibility of reconstruction of dental plaster cast from 3D digital study models," Biomed Eng Online, vol. 12, pp. 49, 2013.

[2] F. F. Al-Jassir, H. Fouad, O. Y. Alothman, "In vitro assessment of Function Graded (FG) artificial Hip joint stem in terms of bone/cement stresses: 3D Finite Element (FE) study," Biomed Eng Online, vol. 12, pp. 5, 2013.

[3] C. E. Dombroski, M. E. Balsdon, and A. Froats, "The use of a low cost 3D scanning and printing tool in the manufacture of custom-made foot orthoses: a preliminary study," BMC Res Notes, vol. 7, pp. 443, 2014.

[4] J. S. Lee, J. M. Hong, J. W. Jung, et al., "3D printing of composite tissue with complex shape applied to ear regeneration," Biofabrication, vol. 6, pp. 024103, 2014.

[5] J. V. Vanore, J. C. Christensen, S. R. Kravitz, et al., "Diagnosis and treatment of first metatarsophalangeal joint disorders. Section 1: Hallux valgus," J. Foot Ankle Surg., vol. 42, pp. 112-23, 2003.

[6] E. M. Roos, S. Brandsson, and J. Karlsson, "Validation of the foot and ankle outcome score for ankle ligament reconstruction," Foot Ankle Int, vol. 22, pp. 788-94, 2001.

[7] P. M. Kluding, K. Dunning, M. W. O'Dell, et al., "Foot drop stimulation versus ankle foot orthosis after stroke: 30week outcomes," Stroke, vol. 44, pp. 1660-9, 2013.

[8] S. F. Tang, C. P. Chen, J. L. Pan, et al., "The effects of a new foot-toe orthosis in treating painful hallux valgus," Arch Phys Med Rehabil, vol. 83, pp. 1792-5, 2002.

[9] R. Schuh, M. Willegger, J. Holinka, et al., "Angular correction and complications of proximal first metatarsal osteotomies for hallux valgus deformity," Int Orthop, vol. 37, pp. 1771-80, 2013.

[10] K. T. Lee, Y. U. Park, H. Jegal, et al., "Osteoarthritis of the second metatarsophalangeal joint associated with hallux valgus deformity," Foot Ankle Int, vol. 35, pp. 1329-33, 2014.

[11] D. Kaya, O. A. Atay, M. J. Callaghan, et al., "Hallux valgus in patients with patellofemoral pain syndrome," Knee Surg Sports Traumatol Arthrosc, vol. 17, pp. 1364-7, 2009.

[12] H. Gong, Z. C. Sang, J. M. Wen, et al., "Correlative analysis on metatarsalgia and the X-ray measurement indexes under weight-bearing and non-weight-bearing of hallux valgus," Zhongguo Gu Shang, vol. 27, pp. 303-7, 2014. 